\title{
Generators and defining equation of the modular function field of the group $\Gamma_{1}(N)$
}

\author{
by \\ Nobuhiko Ishida and Noburo Ishiı (Osaka)
}

1. Introduction. Let $N$ be a positive integer. Let $\Gamma(N)$ denote the principal congruence subgroup of level $N$ and $\Gamma_{1}(N)$ a subgroup of $\mathrm{SL}_{2}(\mathbb{Z})$ defined by

$$
\Gamma_{1}(N)=\left\{\left(\begin{array}{ll}
a & b \\
c & d
\end{array}\right) \in \mathrm{SL}_{2}(\mathbb{Z}) \mid a \equiv d \equiv 1 \bmod N, c \equiv 0 \bmod N\right\} .
$$

Let $A(N)$ and $A_{1}(N)$ be the modular function fields with respect to the groups $\Gamma(N)$ and $\Gamma_{1}(N)$ respectively. Further let $X_{1}(N)$ be the modular curve associated with the modular function field $A_{1}(N)$. The genus of $X_{1}(N)$ is $\geq 1$ if and only if $N=11, N \geq 13$. The purpose of this paper is to construct "good" generators of $A_{1}(N)$ such that we can obtain a "simple" equation of the field $A_{1}(N)$, which gives an affine, in general, singular model over $\mathbb{Q}$ of the curve $X_{1}(N)$.

The non-cuspidal, $\mathbb{C}$-rational points of $X_{1}(N)$ parametrize the isomorphism classes of pairs of the elliptic curve over $\mathbb{C}$ and a point of order $N$ on it. From this property, Reichert [9] obtained the equations of $X_{1}(N)$ for $N=11,13, \ldots, 18$ from "raw forms" which were deduced from the equation satisfied by $N$-torsion points on the elliptic curve called the $E(b, c)$-form. Further he calculated tables of elliptic curves over quadratic fields with torsion groups of special types. Independently, Lecacheux [7], Washington [11] and Darmon [2] constructed generators of the field $A_{1}(N)$ explicitly and determined the equation of $X_{1}(N)$ for $N=13,16,25$ respectively, for the purpose of obtaining a family of cyclic extensions over $\mathbb{Q}$. The authors [3]-[5] constructed generators of $A(N), A_{1}(N)$ for any $N \geq 6$ and showed that the equation of $A_{1}(N)$ can be deduced very easily from the equation of $A(N)$ deduced from the relation between them. However our equation given in [5] is not simple as compared with the "raw forms" of Reichert. In this paper, we construct new kind of generators of $A_{1}(N)$ for any integer greater than 10

2000 Mathematics Subject Classification: Primary 14H05; Secondary 11F03, 11G18. 
from similar functions used by Lecacheux, Washington and Darmon. The equations obtained from these new generators are as simple as the "raw forms" of Reichert.

In Sections 2 and 3, we shall introduce modular functions $W_{3}, W_{4}, W_{5}$ of $\Gamma_{1}(N)$ which are modular units (for modular units, see Kubert and Lang [6]) and show that the pairs $\left(W_{3}, W_{5}\right),\left(W_{3}, W_{4}\right)$ generate $A_{1}(N)$ over $\mathbb{C}$, respectively. In Section 4, we shall study the properties of the equation of $A_{1}(N)$ obtained from the relation between $W_{3}$ and $W_{5}$. In the last part of Section 4 , as examples, we shall give equations for $11 \leq N \leq 20, N \neq 12$. Let $J$ be the modular invariant function. In Section 5 , we shall also show that the pairs $\left(J, W_{3}\right)$ and $\left(J, W_{5}\right)$ of modular functions each generate $A_{1}(N)$ over $\mathbb{C}$.

Throughout this paper, we shall use the following notation. For finitely many elements $a_{1}, \ldots, a_{m}$ of a unique factorization domain, we denote by $\operatorname{GCD}\left(a_{1}, \ldots, a_{m}\right)$ the greatest common divisor of $a_{1}, \ldots, a_{m}$. For $x \in \mathbb{R}$, we denote by $[x]$ the greatest integer not exceeding $x$. For a function $f(\tau)$ on the complex upper half plane and $A=\left(\begin{array}{ll}a & b \\ c & d\end{array}\right) \in \mathrm{SL}_{2}(\mathbb{Z})$, we put

$$
\left.f\right|_{2}[A]=f(A(\tau))(c \tau+d)^{-2}
$$

where $A(\tau)=(a \tau+b) /(c \tau+d)$.

2. The function $W_{r}(\tau)$. Let $N$ be a positive integer greater than 10 . For a complex number $\tau$ in the complex upper half plane, we denote by $L_{\tau}$ the lattice in $\mathbb{C}$ generated by 1 and $\tau$ and by $\wp\left(z ; L_{\tau}\right)$ the Weierstrass $\wp$-function associated with $L_{\tau}$. For a pair $(r, s)$ of integers such that $(r, s) \not \equiv$ $(0,0) \bmod N$, consider the function

$$
E(\tau ; r, s, N)=\wp\left(\frac{r \tau+s}{N} ; L_{\tau}\right)
$$

on the complex upper half plane. Then it is easy to see that $E(\tau ; r, s, N)$ has the following transformation formula:

$$
\left.E(\tau ; r, s, N)\right|_{2}[A]=E(\tau ; a r+c s, b r+d s, N) \quad \text { for } A=\left(\begin{array}{cc}
a & b \\
c & d
\end{array}\right) \in \mathrm{SL}_{2}(\mathbb{Z}) .
$$

In particular, for an integer $s$ not congruent to $0 \bmod N$, we know that the function

$$
\phi_{s}(\tau)=\frac{1}{(2 \pi i)^{2}} \wp\left(\frac{s}{N} ; L_{\tau}\right)=\frac{1}{(2 \pi i)^{2}} E(\tau ; 0, s, N)
$$

is a modular form of weight 2 of the group $\Gamma_{1}(N)$. Further if $r$ and $s$ are integers such that $r \not \equiv \pm s \bmod N$, then $\phi_{r}(\tau)-\phi_{s}(\tau)$ has neither zeros nor poles on the complex upper half plane, because the function $\wp\left(z ; L_{\tau}\right)-$ $\wp\left(s / N ; L_{\tau}\right)$ has zeros (resp. poles) only at the points $z \equiv \pm s / N$ (resp. 0) $\bmod L_{\tau}$. For a positive integer $r$ not congruent to $0, \pm 1, \pm 2 \bmod N$, we define 
a modular function $W_{r}(\tau)$ with respect to $\Gamma_{1}(N)$ by

$$
W_{r}(\tau)=\frac{\phi_{2}(\tau)-\phi_{1}(\tau)}{\phi_{r}(\tau)-\phi_{1}(\tau)} .
$$

The function $W_{r}(\tau)$ has neither zeros nor poles on the complex upper half plane. We shall determine the order of $W_{r}(\tau)$ at the cusps of $\Gamma_{1}(N)$. In Ogg [8], all inequivalent cusps of $\Gamma_{1}(N)$ are given by the pairs $(u, t)$ of integers such that:

- $1 \leq t<N / 2,1 \leq u \leq D, \operatorname{GCD}(u, D)=1$, or

- $t=N / 2, N, 1 \leq u \leq D / 2, \operatorname{GCD}(u, D)=1$,

where $D=\operatorname{GCD}(t, N)$. Let $(u, t)$ be one of the above cusps. Then, since $\operatorname{GCD}(u, t, N)=1$, we can take a matrix $B(u, t) \in \mathrm{SL}_{2}(\mathbb{Z})$ such that

$$
B(u, t)=\left(\begin{array}{ll}
a & b \\
c & d
\end{array}\right) \equiv\left(\begin{array}{ll}
u & * \\
t & *
\end{array}\right) \bmod N
$$

In the following, let $q=\exp (2 \pi i \tau / N)$ and $\zeta=\exp (2 \pi i / N)$. We know that $q^{D}$ is the local parameter at the cusp $(u, t)$. Therefore the order of $W_{r}(\tau)$ at the cusp $(u, t)$ is equal to the order of the $q^{D}$-expansion of $W_{r}(B(u, t)(\tau))$. To describe the order of $W_{r}(\tau)$ at $(u, t)$, we need the following notation. For an integer $s$, we denote by $\{s\}$ and $\mu(s)$ the integers uniquely determined by the following conditions:

$$
0 \leq\{s\} \leq N / 2, \quad \mu(s)= \pm 1, \quad s \equiv \mu(s)\{s\} \bmod N,
$$

and further if $\{s\}=0$ or $N / 2$, then $\mu(s)=1$.

LEMMA 1. The function $\left.\phi_{s}\right|_{2}[B(u, t)]$ has the following q-expansion:

$$
\begin{aligned}
& \left.\phi_{s}\right|_{2}[B(u, t)]-\frac{1}{12} \\
& = \begin{cases}\frac{\zeta^{s^{*}}}{\left(1-\zeta^{*}\right)^{2}}-\sum_{m=1}^{\infty} \sum_{n=1}^{\infty} n\left(1-\zeta^{s^{*} n}\right)\left(1-\zeta^{-s^{*} n}\right) q^{m n N} & \text { if }\{s t\}=0, \\
\sum_{n=1}^{\infty} n \zeta^{s^{*} n} q^{\{s t\} n}+\sum_{m=1}^{\infty} \sum_{n=1}^{\infty} n\left(\zeta^{s^{*} n} q^{\{s t\} n}+\zeta^{-s^{*} n} q^{-\{s t\} n}-2\right) q^{m n N} & \text { otherwise, }\end{cases}
\end{aligned}
$$

where $s^{*}=\mu(s t) s d$.

Proof. Since $\wp\left(z ; L_{\tau}\right)$ is an $L_{\tau}$-invariant even function, we have

$$
\left.\phi_{s}\right|_{2}[B(u, t)]=\frac{1}{(2 \pi i)^{2}} \wp\left(\frac{s t \tau+s d}{N} ; L_{\tau}\right)=\frac{1}{(2 \pi i)^{2}} \wp\left(\frac{\{s t\} \tau+s^{*}}{N} ; L_{\tau}\right) .
$$

The assertion follows from the well known expansion formula for $\wp\left(z ; L_{\tau}\right)$ 
(see Robert [10], II, 5):

$$
\frac{1}{(2 \pi i)^{2}} \wp\left(z ; L_{\tau}\right)=\sum_{m=-\infty}^{\infty} \frac{e^{2 \pi i z} q^{m N}}{\left(1-e^{2 \pi i z} q^{m N}\right)^{2}}+\frac{1}{12}-2 \sum_{m=1}^{\infty} \frac{q^{m N}}{\left(1-q^{m N}\right)^{2}} .
$$

(Use the fact $x /(1-x)^{2}=\sum_{m=1}^{\infty} m x^{m}$.)

Lemma 2. Let $s$ be an integer such that $s \not \equiv 0 \bmod N$. Further, for $N$ odd (resp. even), assume $s \not \equiv \pm 1 \bmod N($ resp. $N / 2)$. Then the order of the $q$-expansion of $\left.\left(\phi_{s}-\phi_{1}\right)\right|_{2}[B(u, t)]$ is $\min (\{s t\},\{t\})$.

Proof. By Lemma 1, we know that the $q$-expansion of $\left.\phi_{s}\right|_{2}[B(u, t)]-$ $1 / 12$ begins with the term:

$$
\begin{cases}\frac{\zeta^{s d}}{\left(1-\zeta^{s d}\right)^{2}} & \text { if }\{s t\}=0 \\ \left(\zeta^{\mu(s t) s d}+\zeta^{-\mu(s t) s d}\right) q^{N / 2} & \text { if } N \text { is even and }\{s t\}=N / 2 \\ \zeta^{\mu(s t) s d} q^{\{s t\}} & \text { otherwise. }\end{cases}
$$

It is to be noted that the coefficient $\left(\zeta^{\mu(s t) s d}+\zeta^{-\mu(s t) s d}\right)$ of $q^{N / 2}$ in the second case can be zero and the coefficients in the other cases are not zero. If $\{s t\} \neq\{t\}$, then we get easily our assertion. Assume $\{s t\}=\{t\}$. We must show that the coefficient $C$ of $q^{\{s t\}}$ of the $q$-expansion of the function $\left.\left(\phi_{s}-\phi_{1}\right)\right|_{2}[B(u, t)]$ is not zero.

First assume $\{s t\}=\{t\}=0$. Then $t=N$ and the coefficient $C$ is

$$
\frac{\zeta^{s d}}{\left(1-\zeta^{s d}\right)^{2}}-\frac{\zeta^{d}}{\left(1-\zeta^{d}\right)^{2}}=-\frac{\zeta^{d}\left(\zeta^{(s-1) d}-1\right)\left(\zeta^{(s+1) d}-1\right)}{\left(1-\zeta^{s d}\right)^{2}\left(1-\zeta^{d}\right)^{2}} .
$$

Since $\operatorname{GCD}(d, N)=1$ and $s \not \equiv \pm 1 \bmod N$, this is not zero.

Next assume $\{s t\}=\{t\}=N / 2$. Then we know $s$ is odd, $t=N / 2$, $\mu(s t)=\mu(t)=1$ and the coefficient $C$ is

$$
\zeta^{s d}+\zeta^{-s d}-\zeta^{d}-\zeta^{-d}=\frac{\left(\zeta^{(s+1) d}-1\right)\left(\zeta^{(s-1) d}-1\right)}{\zeta^{s d}} .
$$

If this is zero, then $(s \pm 1) d \equiv 0 \bmod N$. Since $t=N / 2$, we have $\operatorname{GCD}(d, N / 2)$ $=1$. Therefore, $s \equiv \pm 1 \bmod N / 2$. This contradicts our assumption.

Finally, assume $\{s t\}=\{t\} \neq 0, N / 2$. Then $C=\zeta^{\mu(s t) s d}-\zeta^{\mu(t) d}$. If $C=0$, then $\mu(s t) s d \equiv \mu(t) d \bmod N$. Furthermore, since $\{s t\}=\{t\}$, we have $\mu(s t) s t \equiv \mu(t) t \bmod N$. Since $\operatorname{GCD}(N, t, d)=1$, these two congruences show $s \equiv \pm 1 \bmod N$. This contradicts the assumption.

Since the local parameter at the cusp $(u, t)$ is $q^{D}$, by Lemma 2, we have immediately

Proposition 1. Let $r$ be a positive integer such that $r \not \equiv 0, \pm 1, \pm 2$ $\bmod N$. Further, for $N$ even, assume that $r \not \equiv \pm 1 \bmod N / 2$. Then $W_{r}$ has 
poles or zeros only at the cusps and the order of $W_{r}$ at the cusp $(u, t)$ is

$$
\frac{\min (\{2 t\},\{t\})-\min (\{r t\},\{t\})}{D},
$$

where $D=\operatorname{GCD}(t, N)$. Furthermore $W_{r}$ takes the value 1 at the cusps $(u, t)$ for $t$ such that $t<\{2 t\},\{r t\}$. Note that the order is determined only by $t$ and is independent of $u$.

3. Generators $\left(W_{3}, W_{4}\right),\left(W_{3}, W_{5}\right)$. Let $N \geq 11, N \neq 12$. In this section, we shall show that the pairs $\left(W_{3}, W_{4}\right)$ and $\left(W_{3}, W_{5}\right)$ of functions each generate $A_{1}(N)$ over $\mathbb{C}$. Let us consider the representatives $(u, t)$ of inequivalent cusps of $\Gamma_{1}(N)$ given in Section 1. Since the order of $W_{r}$ at the cusp $(u, t)$ depends only on $t$, we denote it by $\nu_{t}\left(W_{r}\right)$. For a non-negative integer $k$, if $k N / 2 \leq r t<(k+1) N / 2$, then

$$
\{r t\}= \begin{cases}r t-k N / 2 & \text { if } k \text { is even, } \\ (k+1) N / 2-r t & \text { if } k \text { is odd. }\end{cases}
$$

Let $D=\operatorname{GCD}(t, N)$. Then by Proposition 1 we obtain the following:

$$
\begin{aligned}
& \nu_{t}\left(W_{3}\right)= \begin{cases}0 & \text { if } t \leq N / 4, \\
(4 t-N) / D & \text { if } N / 4 \leq t \leq N / 3, \\
(2 N-5 t) / D & \text { if } N / 3 \leq t \leq N / 2, \\
-1 & \text { if } t=N / 2, \\
0 & \text { if } t=N ;\end{cases} \\
& \nu_{t}\left(W_{4}\right)= \begin{cases}0 & \text { if } t \leq N / 5, \\
(5 t-N) / D & \text { if } N / 5 \leq t \leq N / 4, \\
(N-3 t) / D & \text { if } N / 4 \leq t \leq 2 N / 5, \\
(2 t-N) / D & \text { if } 2 N / 5 \leq t \leq N / 2, \\
0 & \text { if } t=N / 2, \\
0 & \text { if } t=N ;\end{cases} \\
& \nu_{t}\left(W_{5}\right)= \begin{cases}0 & \text { if } t \leq N / 6, \\
(6 t-N) / D & \text { if } N / 6 \leq t \leq N / 5, \\
(N-4 t) / D & \text { if } N / 5 \leq t \leq N / 4, \\
0 & \text { if } N / 4 \leq t \leq N / 3, \\
(3 t-N) / D & \text { if } N / 3 \leq t \leq 2 N / 5, \\
(3 N-7 t) / D & \text { if } 2 N / 5 \leq t \leq N / 2, \\
-1 & \text { if } t=N / 2, \\
0 & \text { if } t=N .\end{cases}
\end{aligned}
$$

We find easily that $W_{3}$ has poles only at the cusps $(u, t)$ such that $2 N / 5<$ $t \leq N / 2, W_{4}$ has poles only at the cusps such that $N / 3<t<N / 2$, and $W_{5}$ has poles only at the cusps such that $3 N / 7<t \leq N / 2$. In particular,

$$
W_{5} \text { has poles only at the points where } W_{3} \text { does. }
$$

We shall make use of this property in the following section. 
Theorem 1. Let the notation be as above. Then

$$
A_{1}(N)=\mathbb{C}\left(W_{3}, W_{4}\right)=\mathbb{C}\left(W_{3}, W_{5}\right) .
$$

Proof. Since we can prove $A_{1}(N)=\mathbb{C}\left(W_{3}, W_{4}\right)$ and $A_{1}(N)=\mathbb{C}\left(W_{3}, W_{5}\right)$ in the same way, we shall prove $A_{1}(N)=\mathbb{C}\left(W_{3}, W_{4}\right)$ in detail, and for $A_{1}(N)=\mathbb{C}\left(W_{3}, W_{5}\right)$ we shall only sketch the proof. For a non-constant function $f$ of $A_{1}(N)$, denote by $d(f)$ the total degree of the poles of $f$. Then $d(f)=\left[A_{1}(N): \mathbb{C}(f)\right]$. Therefore if we can find finitely many functions $f_{1}, \ldots, f_{n}$ in $\mathbb{C}\left(W_{3}, W_{4}\right)$ such that $\operatorname{GCD}\left(d\left(f_{1}\right), \ldots, d\left(f_{n}\right)\right)=1$, we will have $A_{1}(N)=\mathbb{C}\left(W_{3}, W_{4}\right)$.

Let us consider the function $W_{3}^{i}+W_{4}^{j}$ for some $(i, j)$. First, we assume $N$ is odd. In this case, we take two pairs of $(i, j)=(4, N-10),(4, N-9)$. Let $(i, j)=(4, N-10)$. Then for $2 N / 5<t<N / 2$,

$$
\begin{aligned}
\nu_{t}\left(W_{4}^{N-10}\right)-\nu_{t}\left(W_{3}^{4}\right) \\
=(N-10)(2 t-N) / D+4(5 t-2 N) / D=2 N\left(t-\frac{N-2}{2}\right) / D \\
\begin{cases}<0 & \text { if } t<(N-1) / 2, \\
=0 & \text { if } t=(N-1) / 2 .\end{cases}
\end{aligned}
$$

Therefore, by (3) and (4) we obtain

$$
\begin{aligned}
d & \left(W_{3}^{4}+W_{4}^{N-10}\right) \\
= & (N-10)\left\{\sum_{N / 3<t \leq 2 N / 5} \frac{3 t-N}{D} \cdot \varphi(D)+\sum_{2 N / 5<t<N / 2} \frac{N-2 t}{D} \cdot \varphi(D)\right\} \\
& -(N-10)\left(N-2 \cdot \frac{N-1}{2}\right)+4\left(5 \cdot \frac{N-1}{2}-2 N\right) \\
= & (N-10) d\left(W_{4}\right)+N .
\end{aligned}
$$

It is noted that $D=(t, N)=1$ for $t=(N-1) / 2$. Let $(i, j)=(4, N-9)$. Then

$$
4(5 t-2 N) / D-(N-9)(N-2 t) / D=2(N+1)\left(t-\frac{N(N-1)}{2(N+1)}\right) / D .
$$

Since we see easily that

$$
\frac{N-3}{2}<\frac{N(N-1)}{2(N+1)}<\frac{N-1}{2}
$$

we deduce similarly

$$
d\left(W_{3}^{4}+W_{4}^{N-9}\right)=(N-9) d\left(W_{4}\right)+N-1 .
$$

Consequently, for $N$ odd we have $\operatorname{GCD}\left(d\left(W_{4}\right), d\left(W_{3}^{4}+W_{4}^{N-10}\right), d\left(W_{3}^{4}+W_{4}^{N-9}\right)\right)=\operatorname{GCD}\left(d\left(W_{4}\right), N, N-1\right)=1$. 
Next, we assume $N$ is even, and $N \geq 16$ for the present. In this case, we take three pairs of $(i, j)=(1, N-2),(6, N-15),(3,(N-14) / 2)$. Firstly, let $(i, j)=(1, N-2)$. Since

$$
(5 t-2 N) / D-(N-2)(N-2 t) / D=(2 N+1)\left(t-\frac{N^{2}}{2 N+1}\right) / D
$$

and

$$
\frac{N-2}{2}<\frac{N^{2}}{2 N+1}<\frac{N}{2}
$$

we obtain

$$
\begin{aligned}
& d\left(W_{3}+W_{4}^{N-2}\right) \\
& =(N-2)\left\{\sum_{N / 3<t \leq 2 N / 5} \frac{3 t-N}{D} \cdot \varphi(D)+\sum_{2 N / 5<t<N / 2} \frac{N-2 t}{D} \cdot \varphi(D)\right\}+\frac{\varphi(N / 2)}{2} \\
& =(N-2) d\left(W_{4}\right)+\frac{\varphi(N / 2)}{2} .
\end{aligned}
$$

Let $(i, j)=(6, N-15)$. Since

$$
6(5 t-2 N) / D-(N-15)(N-2 t) / D=2 N\left(t-\frac{N-3}{2}\right) / D
$$

and $\delta=((N-2) / 2, N)=1($ resp. 2$)$ if $N \equiv 0 \bmod 4($ resp. $N \equiv 2 \bmod 4)$, we obtain

$$
\begin{aligned}
& d\left(W_{3}^{6}+W_{4}^{N-15}\right) \\
& =(N-15) d\left(W_{4}\right) \\
& \quad-(N-15)\left(N-2 \cdot \frac{N-2}{2}\right) / \delta+6\left(5 \cdot \frac{N-2}{2}-2 N\right) / \delta+6 \cdot \frac{\varphi(N / 2)}{2} \\
& =(N-15) d\left(W_{4}\right)+\frac{N}{\delta}+6 \cdot \frac{\varphi(N / 2)}{2} .
\end{aligned}
$$

Lastly, take $(i, j)=(3,(N-14) / 2)$. Then

$$
3(5 t-2 N) / D-\frac{N-14}{2}(N-2 t) / D=(N+1)\left(t-\frac{N(N-2)}{2(N+1)}\right) / D .
$$

Since

$$
\frac{N-4}{2}<\frac{N(N-2)}{2(N+1)}<\frac{N-2}{2},
$$

we conclude similarly that

$$
d\left(W_{3}^{3}+W_{4}^{(N-14) / 2}\right)=\frac{N-14}{2} d\left(W_{4}\right)+\frac{N-2}{2 \delta}+3 \cdot \frac{\varphi(N / 2)}{2} .
$$


Consequently,

$$
\begin{array}{r}
\operatorname{GCD}\left(d\left(W_{4}\right), d\left(W_{3}+W_{4}^{N-2}\right), d\left(W_{3}^{6}+W_{4}^{N-15}\right), d\left(W_{3}^{3}+W_{4}^{(N-14) / 2}\right)\right) \\
=\operatorname{GCD}\left(d\left(W_{4}\right), \frac{\varphi(N / 2)}{2}, \frac{N}{\delta}, \frac{N-2}{2 \delta}\right)=1 .
\end{array}
$$

For the remaining case of $N=14$, we have $\operatorname{GCD}\left(d\left(W_{4}\right), d\left(W_{3}+W_{4}^{12}\right)\right)$ $=1$. This completes the proof of $A_{1}(N)=\mathbb{C}\left(W_{3}, W_{4}\right)$.

To prove $A_{1}(N)=\mathbb{C}\left(W_{3}, W_{5}\right)$, we may take $(i, j)=(N-14, N-10)$ and $((N-13) / 2,(N-9) / 2)$ for $N$ odd, and $(i, j)=(N-3, N-2)$, $(N-21, N-15)$ and $((N-20) / 2,(N-14) / 2)$ for $N$ even.

4. The defining equation of $A_{1}(N)$. We shall study the minimal equation of $W_{5}$ over $\mathbb{C}\left(W_{3}\right)$, which is a defining equation of $A_{1}(N)$ and gives an affine model of the curve $X_{1}(N)$. To simplify the notation, we write $d_{r}$ instead of $d\left(W_{r}\right)$. Since $W_{3}, W_{5}$ have $q$-expansions at the cusp $i \infty$ with $\mathbb{Q}(\zeta)$-coefficients and $\left[A_{1}(N): \mathbb{C}\left(W_{3}\right)\right]=d_{3}$, the minimal equation $F_{N}\left(W_{3}, Y\right)=0$ of $W_{5}$ over $\mathbb{C}\left(W_{3}\right)$ can be of the form

$$
F_{N}(X, Y)=\Phi_{d_{3}}(X) Y^{d_{3}}+\Phi_{d_{3}-1}(X) Y^{d_{3}-1}+\ldots+\Phi_{1}(X) Y+\Phi_{0}(X),
$$

where $\Phi_{j}(X) \in \mathbb{Q}(\zeta)[X]$ for all $j$, the leading coefficient of $\Phi_{d_{3}}(X)$ is equal to 1 , and $\Phi_{d_{3}}(X), \ldots, \Phi_{1}(X)$ and $\Phi_{0}(X)$ have no common factors except nonzero constants. Because we shall use a similar argument to that in Section 3 of Ishida and Ishii [4], we shall be brief. For details see [4]. Assume $F$ and $G$ generate $A_{1}(N)$ over $\mathbb{C}$, that is, $A_{1}(N)=\mathbb{C}(F, G)$. Let $\Phi(X, Y) \in \mathbb{C}[X, Y]$ be the polynomial such that $\Phi(F, Y)=0$ is the minimal equation of $G$ over $\mathbb{C}$. It has degree $d=d(F)$ as a polynomial of $Y$. Let $R_{1}$ denote the Riemann surface associated with $A_{1}(N)$. Then the inclusion of $\mathbb{C}(F)$ into $A_{1}(N)$ induces a morphishm $\varphi$ of $R_{1}$ onto the projective space $\mathbb{P}^{1}(\mathbb{C})$ of dimension 1 such that

$$
\varphi(Q)= \begin{cases}{[F(Q), 1]} & \text { if } F(Q) \neq \infty \\ {[1,0]} & \text { otherwise }\end{cases}
$$

For every point $\alpha \in \mathbb{P}^{1}(\mathbb{C})$, its inverse image $\varphi^{*}(\alpha)$ under $\varphi$ is a divisor on $R_{1}$ given by

$$
\varphi^{*}(\alpha)=\sum_{i=1}^{M} e_{i} Q_{i},
$$

where $Q_{i}$ are all the distinct points of $R_{1}$ such that $F\left(Q_{i}\right)=\alpha$ and $e_{i}$ is the absolute value of the order of $F$ at the point $Q_{i}$. Let $T$ be an indeterminate and $\mathbb{C}[[T]]$ the ring of formal power series in $T$ and $\mathbb{C}((T))$ its fractional field. Put $U=T+\alpha($ resp. $1 / T)$ if $\alpha \neq \infty$ (resp. $\alpha=\infty)$. We can write 
$\Phi(U, Y)=h(T) \Psi(Y)$, where

$$
\begin{aligned}
& h(T) \in \mathbb{C}((T)), \\
& \Psi(Y)=T^{m} Y^{d}+\Psi_{d-1}(T) Y^{d-1}+\ldots+\Psi_{1}(T) Y+\Psi_{0}(T),
\end{aligned}
$$

$m$ is a non-negative integer and $\Psi_{j}(T) \in \mathbb{C}[[T]]$ for all $j$. Further if $m \geq 1$ then at least one of $\Psi_{j}(T)(0 \leq j \leq d-1)$ is not divisible by $T$. By $(7)$, we know that $\Psi(Y)$ decomposes into a product of $M$ irreducible polynomials $G_{i}(Y)$ of degree $e_{i}$ with coefficients in $\mathbb{C}[[T]]$. Let || be a valuation on $\mathbb{C}((T))$ defined by $|T|=\lambda$ for a $\lambda \in \mathbb{R}, 0<\lambda<1$. Let $f_{i}$ be the order of $G$ at the point $Q_{i}$. Then we know that $G_{i}(Y)$ is pure of type $\left(e_{i},-\left(f_{i} / e_{i}\right) \log \lambda\right)$. Further if we put

$$
G_{i}(Y)=g_{i, e_{i}}(T) Y^{e_{i}}+\ldots+g_{i, 1}(T) Y+g_{i, 0}(T),
$$

where $g_{i, k}(T) \in \mathbb{C}[[T]]$ for all $k$ and $\operatorname{GCD}\left(g_{i, e_{i}}(T), \ldots, g_{i, 0}(T)\right)=1$, then (2.6) of [4] gives

(8) $G$ has a pole (resp. zero) at $Q_{i}$ if and only if

$$
\left|g_{i, e_{i}}(T)\right|\left(\operatorname{resp} .\left|g_{i, 0}(T)\right|\right)<1 \text {. }
$$

LEMMA 3. Let the notation be as above. Assume that the coefficients of $\Phi(X, Y)$ as a polynomial of $Y$ have no common factors. Let $P_{1}, \ldots, P_{m}$ be all the distinct points of $R_{1}$ where $F$ has zeros. Let $e_{i}$ be the order of the zero of $F$ at $P_{i}$. Further assume that $G$ takes the value $\infty, 0,1$ at $P_{i}$ for $1 \leq i \leq k, k+1 \leq i \leq l, l+1 \leq i \leq m$ respectively. Then

$$
\Phi(0, Y)=c^{*} Y^{a}(Y-1)^{b},
$$

where $c^{*}$ is a non-zero constant and

$$
a=\sum_{k+1 \leq i \leq l} e_{i}, \quad b=\sum_{l+1 \leq i \leq m} e_{i} .
$$

Proof. Write $\Phi(T, Y)=h(T) \Psi(Y)$ as above. Then by assumption $h(0)$ $\neq 0$. Decompose $\Psi(Y)$ into irreducible factors $G_{i}(Y)$ which correspond to $P_{i}$. Put

$$
G_{i}(Y)=g_{i, e_{i}}(T) Y^{e_{i}}+\ldots+g_{i, 1}(T) Y+g_{i, 0}(T)
$$

By assumption and (8), we have:

- if $1 \leq i \leq k$, then

$$
\left|g_{i, 0}(T)\right|=1, \quad\left|g_{i, j}(T)\right|<1 \quad \text { for } j \neq 0,
$$

- if $k+1 \leq i \leq l$, then

$$
\left|g_{i, e_{i}}(T)\right|=1, \quad\left|g_{i, j}(T)\right|<1 \quad \text { for } j \neq e_{i} .
$$

For $l+1 \leq i \leq m$, since $G$ takes the value $1, G_{i}(Y)$ is written as a polynomial of $Y-1$ in

$$
G_{i}(Y)=g_{i, e_{i}}^{*}(T)(Y-1)^{e_{i}}+\ldots+g_{i, 1}^{*}(T)(Y-1)+g_{i, 0}^{*}(T),
$$


where $\left|g_{i, e_{i}}^{*}(T)\right|=1,\left|g_{i, j}^{*}(T)\right|<1$ for $j \neq e_{i}$. Since, for any power series $\omega(T) \in \mathbb{C}[[T]]$,

$$
|\omega(T)|<1 \quad \text { if and only if } \quad \omega(0)=0,
$$

we have

$$
\begin{aligned}
\Phi(0, Y) & =h(0) \prod_{1 \leq i \leq k} g_{i, 0}(0) \prod_{k+1 \leq i \leq l} g_{i, e_{i}}(0) Y^{e_{i}} \prod_{l+1 \leq i \leq m} g_{i, e_{i}}^{*}(0)(Y-1)^{e_{i}} \\
& =c^{*} Y^{a}(Y-1)^{b} .
\end{aligned}
$$

In the following, to simplify the notation, we write $g_{i, k}$ instead of $g_{i, k}(T)$ if it is unnecessary to say explicitly that $g_{i, k}(T)$ is a power series of $T$.

TheOREM 2. Let the assumption and the notation be as above, and let $N \geq 11, \neq 12$. Then:

(i) $\Phi_{d_{3}}(X)=1$.

(ii) $\max _{0 \leq j \leq d_{3}} \operatorname{deg} \Phi_{j}(X)=d_{5}$. Furthermore, if $7 \nmid N$, then

$$
\operatorname{deg} \Phi_{j}(X)<\operatorname{deg} \Phi_{a}(X)=d_{5} \quad \text { for all } j \neq a,
$$

where

$$
a=\sum_{2 N / 5<t<3 N / 7} \frac{5 t-2 N}{D} \cdot \varphi(D) \quad \text { and } \quad D=\operatorname{GCD}(t, N) .
$$

(iii) If $N$ is odd, then

$$
\operatorname{deg} \Phi_{j}(X) \leq \min \left(d_{5}, \frac{(N-7)\left(d_{3}-j\right)}{N-5}\right) .
$$

If $N$ is even, then

$$
\operatorname{deg} \Phi_{j}(X) \leq \min \left(d_{5}, d_{3}-j\right) .
$$

(iv) $\Phi_{j}(X) \in \mathbb{Q}[X]$ for all $j$.

Proof. Let $(F, G)=\left(W_{3}, W_{5}\right)$ in the above explanation. By $(6), W_{5}$ has poles only at the points where $W_{3}$ does. Therefore the same argument as in Lemma 2 of [4] shows (i).

Next we prove (ii). By applying the latter part of Lemma 3 of [4] to the functions $W_{3}, W_{5}$ and the polynomial $F_{N}(X, Y)$, we obtain, by (i), $\max _{j} \operatorname{deg} \Phi_{j}(X)=d_{5}$. Let $\alpha=\infty$ and consider the decomposition

$$
\Psi(Y)=T^{d_{5}} F_{N}(1 / T, Y)=\prod_{t} G_{(u, t)}(Y) .
$$

Here $G_{(u, t)}(Y)$ are the irreducible factors corresponding to the cusps $(u, t)$ where $W_{3}$ has poles, thus, the product runs through all the cusps $(u, t)$ such that

$2 N / 5<t \leq N / 2$, and if $t<N / 2($ resp. $t=N / 2)$, then $1 \leq u \leq D$ $($ resp. $1 \leq u \leq D / 2), \operatorname{GCD}(u, D)=1$. 
Since the degree of $G_{(u, t)}$ and the order of $W_{5}$ at the cusp $(u, t)$ depend only on $t$ by Proposition 1, we denote them by $e_{t}$ and $f_{t}$ respectively. Now let $7 \nmid N$. Since $W_{5}$ has zeros (resp. poles) at the cusp $(u, t)$ for $2 N / 5<t<3 N / 7$ (resp. $3 N / 7<t \leq N / 2$ ), by (8) and (2.6) of [4], for the coefficients $g_{(u, t), j}$ of $G_{(u, t)}$, we have

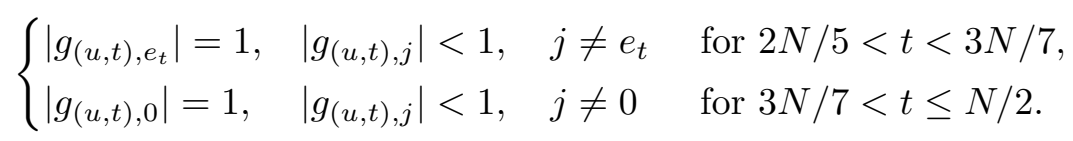

Therefore

$$
\begin{aligned}
T^{d_{5}} F_{N}(1 / T, Y)= & \left(\prod_{2 N / 5<t<3 N / 7} \prod_{u} g_{(u, t), e_{t}} \prod_{3 N / 7<t \leq N / 2} \prod_{u} g_{(u, t), 0}\right) Y^{a} \\
& +T H(Y),
\end{aligned}
$$

where $H(Y)$ is an element of $\mathbb{C}[[T]](Y)$ and for each $t$, the $u$-product runs over all integers $u$ such that the pair $(u, t)$ satisfies (9). This shows (ii).

To prove (iii), let $\alpha=\infty$ again. By $(5), G_{(u, t)}(Y)$ is pure of type $\left(e_{t}, \gamma_{t}\right)$, where

$$
e_{t}=5 t-2 N, \quad \gamma_{t}=-\frac{f_{t}}{e_{t}} \log \lambda=\frac{7 t-3 N}{5 t-2 N} \log \lambda .
$$

Since $\gamma_{t}<\gamma_{t^{\prime}}$ for $t>t^{\prime}, \gamma_{(N-1) / 2}\left(\right.$ resp. $\left.\gamma_{N / 2}\right)$ is the smallest slope among $\gamma_{t}$ 's if $N$ is odd (resp. even). Put

$$
c= \begin{cases}\exp \left(-\gamma_{(N-1) / 2}\right) & \text { if } N \text { is odd } \\ \exp \left(-\gamma_{N / 2}\right) & \text { if } N \text { is even. }\end{cases}
$$

Further extend the valuation || to the valuation \|\|$_{c}$ of $\mathbb{C}((T))(Y)$. See Cassels [1] for the definition of \|\|$_{c}$. Then, from the choice of $c$, we know that $\log \left(\left|g_{(u, t), e_{t}}\right| c^{e_{t}}\right) \geq \log \left(\left|g_{(u, t), j}\right| c^{j}\right)$ for all $j$. Thus, by (10), we have

$$
\begin{aligned}
\max _{j}\left(\left|\Phi_{j}\left(\frac{1}{T}\right) T^{d_{5}}\right| c^{j}\right) & =\|\Psi(Y)\|_{c}=\prod\left\|G_{(u, t)}(Y)\right\|_{c}=\prod\left|g_{(u, t), e_{t}}\right| c^{e_{t}} \\
& =\lambda^{d_{5}} c^{d_{3}} .
\end{aligned}
$$

This shows

$$
\lambda^{d_{5}-\operatorname{deg} \Phi_{j}(X)} c^{j} \leq \lambda^{d_{5}} c^{d_{3}} .
$$

By taking log on both sides, we have (iii).

To prove (iv), we shall transform $W_{3}$ and $W_{5}$ by the Atkin-Lehner involution. Put $V_{3}(\tau)=W_{3}(-1 /(N \tau))$ and $V_{5}(\tau)=W_{5}(-1 /(N \tau))$. Note that $A_{1}(N)=\mathbb{C}\left(V_{3}, V_{5}\right)$ and $F_{N}\left(V_{3}, Y\right)=0$ is the minimal equation of $V_{5}$ over $\mathbb{C}\left(V_{3}\right)$. By the definition of the form $\phi_{s}(\tau)$ and the transformation formula for $E(\tau ; r, s, N)$, we have

$$
\phi_{s}\left(\frac{-1}{N \tau}\right)(N \tau)^{-2}=\frac{1}{(2 \pi i)^{2}} \wp\left(s \tau, L_{N \tau}\right) .
$$


Furthermore by the expansion formula for the $\wp$-function given in Lemma 1, $\frac{1}{(2 \pi i)^{2}} \wp\left(s \tau, L_{N \tau}\right)$ has a $q$-expansion with $\mathbb{Q}$-coefficients. Thus the $q$-expansion of $V_{r}$ lies in $\mathbb{Q}((q))$. Let us extend any element $\sigma \in \operatorname{Gal}(\mathbb{Q}(\zeta) / \mathbb{Q})$ to an automorphism of $\mathbb{Q}(\zeta)((q))$ by the mapping $\sum c_{n} q^{n} \mapsto \sum c_{n}^{\sigma} q^{n}$. Then, since $V_{r}^{\sigma}=V_{r}(r=3,5)$, we have

$$
F_{N}\left(V_{3}, V_{5}\right)^{\sigma}=F_{N}^{\sigma}\left(V_{3}^{\sigma}, V_{5}^{\sigma}\right)=F_{N}^{\sigma}\left(V_{3}, V_{5}\right)=0 .
$$

This implies that $F_{N}^{\sigma}\left(V_{3}, Y\right)=0$ is the minimal equation of $V_{5}$. Thus we have $F_{N}^{\sigma}(X, Y)=F_{N}(X, Y)$. Hence $\Phi_{j}(X) \in \mathbb{Q}[X]$ for all $j$.

From Lemma 3 we obtain some properties of $F_{N}(X, Y)$.

TheORem 3. Let the notation be as above. Further assume $N$ is prime. Then:

(i) $F_{N}(0, Y)=F_{N}(1, Y)=Y^{\alpha}(Y-1)^{d_{3}-\alpha}$.

(ii) $F_{N}(X, 0)=c_{1} X^{\beta}(X-1)^{\gamma-\beta}$.

(iii) $F_{N}(X, 1)=c_{2} X^{\delta}(X-1)^{d_{5}-\delta}$.

(iv) $F_{N}(X, X)=X^{\varepsilon}(X-1)^{d_{3}-\varepsilon}$.

Here $c_{1}, c_{2}$ are non-zero constants and

$$
\begin{aligned}
\alpha & =\sum_{N / 3<t<2 N / 5}(2 N-5 t), \quad \beta=\sum_{N / 3<t<2 N / 5}(3 t-N), \\
\gamma & =\sum_{2 N / 5<t<N / 2}(7 t-3 N), \\
\delta & =\sum_{N / 4<t<2 N / 7}(4 t-N)+\sum_{2 N / 7<t<N / 3}(N-3 t), \\
\varepsilon & =\sum_{N / 3<t<3 N / 8}(3 t-N)+\sum_{3 N / 8<t<2 N / 5}(2 N-5 t) .
\end{aligned}
$$

Proof. The assertions for $F_{N}(0, Y), F_{N}(X, 0)$ are obtained from Proposition $1,(3)$ and (5) by applying Lemma 3 to the pairs $(F, G)=\left(W_{3}, W_{5}\right)$ and $(F, G)=\left(W_{5}, W_{3}\right)$ respectively.

Next we shall prove the assertion for $F_{N}(1, Y)$. Consider the function

$$
V=W_{3}-1=\frac{\phi_{2}-\phi_{3}}{\phi_{3}-\phi_{1}} \text {. }
$$

Then all the points where $V$ has zeros are the cusps $(1, t)$ for $t<N / 4$. Further for $t<N / 4, W_{5}$ takes the value 1 (resp. 0) for $t<N / 6$ (resp. $N / 6<t<N / 4)$. Put $Z=X-1$ and $\Psi(Z, Y)=F_{N}(Z+1, Y)$. Then $\Psi(V, Y)=0$ is the minimal equation of $W_{5}$ over $\mathbb{C}(V)$. Apply Lemma 3 to $(F, G)=\left(V, W_{5}\right)$. Because the order of $V$ at $(1, t)$ is $t$ for $t<N / 6$ and 
$d(V)=d_{3}$, we see that

$$
F_{N}(1, Y)=\Psi(0, Y)=Y^{d_{3}-h}(Y-1)^{h},
$$

where $h=\sum_{t<N / 6} t$. Furthermore it is easy to see that

$$
h=\sum_{N / 4<t<N / 3}(4 t-N)=d_{3}-\alpha .
$$

This completes the proof of (i). Applying Lemma 3 to $(F, G)=\left(W_{5}-1, W_{3}\right)$, similarly, we can show (iii).

Finally, we prove (iv). Let $V_{1}=W_{5}-W_{3}$. Since

$$
V_{1}=\frac{\left(\phi_{2}-\phi_{1}\right)\left(\phi_{3}-\phi_{5}\right)}{\left(\phi_{5}-\phi_{1}\right)\left(\phi_{3}-\phi_{1}\right)}
$$

by (3) and (5) all points where $V_{1}$ has zeros (resp. poles) are the cusps $(1, t)$ for $t<N / 6, N / 3<t<2 N / 5$ (resp. $t>2 N / 5$ ) and

$$
\nu_{t}\left(V_{1}\right)= \begin{cases}3 t-N & \text { if } N / 3<t<3 N / 8, \\ 2 N-5 t & \text { if } t>3 N / 8\end{cases}
$$

Since $A_{1}(N)=\mathbb{C}\left(V_{1}, W_{3}\right), d\left(V_{1}\right)=d_{3}$ and $G(X, Z)=F(X, Z+X)$ is a polynomial of $X$ of degree $d_{3}, G\left(X, V_{1}\right)=0$ is the minimal equation of $W_{3}$ over $\mathbb{C}\left(V_{1}\right)$. The function $W_{3}$ takes the value 1 (resp. 0 ) at the cusps $(1, t)$ for $t<N / 6$ (resp. $N / 3<t<2 N / 5)$. If we apply Lemma 3 to $(F, G)=\left(V_{1}, W_{3}\right)$, we have

$$
F(X, X)=G(X, 0)=X^{\varepsilon}(X-1)^{d_{3}-\varepsilon}
$$

Let $N$ be a prime. Since $d_{3}$ is also equal to the total degree of zeros of $W_{3}$, we have, by $(3)$,

$$
d_{3}=\alpha+\sum_{N / 4<t<N / 3}(4 t-N) .
$$

Thus $0<\alpha<d_{3}$ and $F_{N}(0,0)=F_{N}(1,1)=F_{N}(1,0)=F_{N}(0,1)=0$. From this, the polynomials $F_{N}(X, X), F_{N}(0, X), F_{N}(X, 0)$, and $F_{N}(X, 1)$ are each divisible by $X(X-1)$. Put

$$
\begin{aligned}
& R(X)=\frac{F_{N}(X, X)-F_{N}(0, X)-F_{N}(X, 0)}{X(X-1)}, \\
& S(X)=\frac{F_{N}(X, 0)-F_{N}(X, 1)}{X-1} .
\end{aligned}
$$

Then Theorem 3 yields

Proposition 2. Let $N$ be a prime. Then the polynomial $F_{N}(X, Y)$ can be written in the form: 


$$
\begin{aligned}
F_{N}(X, Y)= & F_{N}(X, X)+F_{N}(0, Y)-F_{N}(0, X) \\
& +(Y-X)\{(Y+X-1) R(X)+Y S(X)\} \\
& +X(X-1) Y(Y-1)(Y-X) U(X, Y),
\end{aligned}
$$

where $U(X, Y) \in \mathbb{Q}[X, Y]$.

Proof. This is obtained by simple computation. We omit the proof.

We can generalize the results of Theorem 3 to $N$ composite as follows.

Theorem 4. (i) If $3 \nmid N$, then $F_{N}(0, Y)=Y^{\alpha}(Y-1)^{d_{3}-\alpha}$.

(ii) If $6 \nmid N$, then $F_{N}(1, Y)=Y^{\alpha^{\prime}}(Y-1)^{d_{3}-\alpha^{\prime}}$.

(iii) If $5 \nmid N$, then $F_{N}(X, 0)=c_{1} X^{\beta}(X-1)^{\gamma}$.

(iv) $F_{N}(X, 1)=c_{2} X^{\delta}(X-1)^{d_{5}-\delta}$.

(v) $F_{N}(X, X)=c_{3} X^{\varepsilon}(X-1)^{d_{3}-\varepsilon}$.

Here $c_{1}, c_{2}$ and $c_{3}$ are non-zero constants and

$$
\begin{aligned}
\alpha & =\sum_{N / 3<t<2 N / 5}((2 N-5 t) / D) \varphi(D), \\
\alpha^{\prime} & =\sum_{N / 6<t \leq N / 5}(t / D) \varphi(D)+\sum_{N / 5<t \leq N / 4}((N-4 t) / D) \varphi(D), \\
\beta & =\sum_{N / 3<t<2 N / 5}((3 t-N) / D) \varphi(D), \\
\gamma & =\sum_{N / 6<t \leq N / 5}((6 t-N) / D) \varphi(D)+\sum_{N / 5<t<N / 4}((N-4 t) / D) \varphi(D), \\
\delta & =\sum_{N / 4<t \leq 2 N / 7}((4 t-N) / D) \varphi(D)+\sum_{2 N / 7<t<N / 3}((N-3 t) / D) \varphi(D), \\
\varepsilon & =\sum_{N / 3<t \leq 3 N / 8}((3 t-N) / D) \varphi(D)+\sum_{3 N / 8<t<2 N / 5}((2 N-5 t) / D) \varphi(D) .
\end{aligned}
$$

Proof. The proof is the same as in the case of $N$ prime so we omit it.

Finally we give some examples.

EXAMPLE. (I) $N$ prime:

$$
\begin{aligned}
F_{11}(X, Y)= & Y^{2}(Y-1)-X(X-1) \\
F_{13}(X, Y)= & Y(Y-1)^{3}+X(X-1) Y+X^{2}(X-1) . \\
F_{17}(X, Y)= & Y^{4}(Y-1)^{3}-4 X(X-1) Y^{4}-X(X-1)(X-10) Y^{3} \\
& +3\left(X^{4}-X^{3}-3 X^{2}+3 X\right) Y^{2} \\
& -\left(X^{5}-5 X^{2}+4 X\right) Y+X(X-1)^{2} .
\end{aligned}
$$




$$
\begin{aligned}
F_{19}(X, Y)= & Y^{3}(Y-1)^{6}+4 X(X-1) Y^{6}-5 X(X-1)(X-2) Y^{5} \\
& -3 X(X-1)\left(X^{2}-5 X-3\right) Y^{4} \\
& +X(X-1)\left(4 X^{3}+X^{2}-16 X-3\right) Y^{3} \\
& -X^{2}(X-1)\left(X^{3}+2 X^{2}+3 X-9\right) Y^{2} \\
& +3 X^{2}(X-1)^{2} Y+X^{2}(X-1)^{3} .
\end{aligned}
$$

(II) $N$ composite:

$$
\begin{aligned}
F_{14}(X, Y)= & Y^{4}-(X+1) Y^{3}-\left(2 X^{2}-3 X\right) Y^{2}+\left(X^{3}-X\right) Y+X(X-1)^{2} . \\
F_{15}(X, Y)= & Y^{5}-3 Y^{4}-3(X-2) Y^{3}+(6 X-7) Y^{2} \\
& +(X-1)\left(2 X^{2}-X-4\right) Y-(X-1)^{2}\left(X^{2}+X+1\right) . \\
F_{16}(X, Y)= & Y^{5}+(2 X-4) Y^{4}-\left(X^{2}+4 X-6\right) Y^{3}+(4 X-4) Y^{2} \\
& +\left(X^{2}-2 X+1\right) Y+X(X-1)^{2} . \\
F_{18}(X, Y)= & Y^{5}-3 Y^{4}-\left(X^{2}-X-\frac{10}{3}\right) Y^{3}+\left(\frac{1}{3} X^{3}+X^{2}-\frac{4}{3} X-\frac{5}{3}\right) Y^{2} \\
& -\left(\frac{2}{3} X^{3}-\frac{1}{3} X^{2}-\frac{1}{3} X-\frac{1}{3}\right) Y+\frac{1}{3} X^{3}(X-1) . \\
F_{20}(X, Y)= & Y^{7}-(3 X+2) Y^{6}+\left(X^{2}+8 X+1\right) Y^{5}-10 X Y^{4} \\
& -\left(5 X^{2}-10 X\right) Y^{3}-\left(2 X^{3}-10 X^{2}+9 X\right) Y^{2} \\
& +\left(2 X^{4}-2 X^{3}-4 X^{2}+4 X\right) Y-X(X-1)^{2}\left(X^{2}+1\right) .
\end{aligned}
$$

Comparing our result with Reichert's [9], our equations seem to correspond to "raw forms" of Reichert.

5. Generators $\left(J, W_{3}\right),\left(J, W_{5}\right)$. Let $J$ be the modular invariant function. We shall show that the pairs $\left(J, W_{3}\right)$ and $\left(J, W_{5}\right)$ are generators of $A_{1}(N)$ over $\mathbb{C}$.

Theorem 5. Let $N=11$ or be an integer $\geq 13$. Then $A_{1}(N)=\mathbb{C}\left(J, W_{3}\right)$ $=\mathbb{C}\left(J, W_{5}\right)$.

Proof. Let $r=3,5$. We know that $A(N)$ is a Galois extension over $\mathbb{C}(J)$ with Galois group $\mathrm{SL}_{2}(\mathbb{Z}) / \pm \Gamma(N)$ and $A_{1}(N)$ is the invariant field associated with the subgroup $\pm \Gamma_{1}(N) / \pm \Gamma(N)$. Therefore to prove $A_{1}(N)=$ $\mathbb{C}\left(J, W_{r}\right)$, it is sufficient to show that for $A \in \mathrm{SL}_{2}(\mathbb{Z}), W_{r}(A(\tau))=W_{r}(\tau)$ implies $A \in \Gamma_{1}(N)\{ \pm 1\}$. Let $A=\left(\begin{array}{ll}a & b \\ c & d\end{array}\right) \in \mathrm{SL}_{2}(\mathbb{Z})$ be such that $W_{r}(A(\tau))=$ $W_{r}(\tau)$.

First of all, we show that $c$ is divisible by $N$. Assume that $c \not \equiv 0 \bmod N$. Without loss of generality, we can regard the matrix $A$ as one of the matrices $B(u, t)$ given by $(2)$ with $c \equiv t \bmod N$. Let $C_{r}$ be the constant term of the $q$-extension of $W_{r}$. By Lemma 2 ,

$$
C_{r}=\frac{\left(\zeta^{r}-1\right)^{2}\left(\zeta^{3}-1\right)(\zeta-1)}{\left(\zeta^{r+1}-1\right)\left(\zeta^{r-1}-1\right)\left(\zeta^{2}-1\right)^{2}} \neq 0 .
$$


Proposition 1 shows that the order of the $q$-extension of $W_{r}(A(\tau))$ is $\min (\{2 c\},\{c\})-\min (\{r c\},\{c\})$. Since $W_{r}(A(\tau))=W_{r}(\tau)$, we see that

$$
\min (\{2 c\},\{c\})=\min (\{r c\},\{c\})
$$

and the coefficient $L_{r}$ of the leading term of $W_{r}(A(\tau))$ is equal to $C_{r}$.

First consider the case $\{2 c\}=\{c\}$. Then $3 c \equiv 0 \bmod N$ and $3 \mid N$. Thus $\{c\}=\{2 c\}=N / 3, \mu(2 c)=-\mu(c),\{3 c\}=0,\{5 c\}=N / 3, \mu(5 c)=-\mu(c)$. Therefore for $r=3$ we have a contradiction. Let $r=5$. Since, in Lemma 2, we know that the coefficient of the leading term of the function $\left.\left(\phi_{s}-\phi_{1}\right)\right|_{2}[A]$ is $\zeta^{\mu(s c) s d}-\zeta^{\mu(c) d}$ in the case $\{c\}=\{s c\} \neq 0, N / 2$ (line 19 in the proof of Lemma 2), we have

$$
L_{5}=\frac{\zeta^{\mu(2 c) 2 d}-\zeta^{\mu(c) d}}{\zeta^{\mu(5 c) 5 d}-\zeta^{\mu(c) d}}=\frac{1}{1+\zeta^{-\mu(c) 3 d}} .
$$

Since $L_{5}=C_{5}$, we have $\left|1 / C_{5}-1\right|=1$. Replacing $C_{5}$ by the value given by (11) for $r=5$, we get

$$
\begin{aligned}
& \left|1 / C_{5}-1\right|^{2} \\
& =\frac{\left(\zeta^{6}+\zeta^{5}+\zeta^{4}+\zeta^{3}+\zeta^{2}+\zeta+1\right)\left(\zeta^{-6}+\zeta^{-5}+\zeta^{-4}+\zeta^{-3}+\zeta^{-2}+\zeta^{-1}+1\right)}{\left(\zeta^{4}+\zeta^{3}+\zeta^{2}+\zeta+1\right)^{2}\left(\zeta^{-4}+\zeta^{-3}+\zeta^{-2}+\zeta^{-1}+1\right)^{2}} \\
& =1 .
\end{aligned}
$$

Since this equation is symmetric with respect to $\zeta$ and $\zeta^{-1}$, after some elementary computation, we can obtain the following equation for $\xi=\zeta+$ $\zeta^{-1}$ :

$$
\xi^{8}+4 \xi^{7}+\xi^{6}-10 \xi^{5}-2 \xi^{4}+14 \xi^{3}-8 \xi=0 .
$$

However, since the irreducible equation of $\xi$ over $\mathbb{Q}$ has degree $\varphi(N) / 2$, we have a contradiction for $N$ such that $\varphi(N) / 2>7$. Further for $N$ such that $\varphi(N) / 2 \leq 7$, by direct computation, we can show $\xi$ does not satisfy the above equation. Thus we also have a contradiction.

Next consider the case $\{2 c\}>\{c\}$. Then $\{c\}=\min (\{r c\},\{c\})$, thus $\{r c\} \geq\{c\}$. If $\{r c\}>\{c\}$, then we have $C_{r}=1$. From this, we have an equation for $\zeta$, but we see immediately that $\zeta$ cannot satisfy it. Assume $\{r c\}=\{c\}$. If $\{c\}=N / 2$, then $\{2 c\}=0<\{c\}$. This contradicts the assumption. If $\{c\}<N / 2$, then by Lemma 2 ,

$$
L_{r}=\frac{-\zeta^{\mu(c) d}}{\zeta^{\mu(r c) r d}-\zeta^{\mu(c) d}} .
$$

Thus $\left|1 / C_{r}-1\right|=1$. Arguing as above, we get a contradiction.

Finally, consider the case $\{2 c\}<\{c\}$. Then we must have $\{r c\}=\{2 c\}$. If $\{2 c\}=0$, we have $\{c\}=0$, because $r$ is odd. If $\{2 c\}=N / 2$, then $\{2 c\} \geq\{c\}$. 
Therefore $\{r c\}=\{2 c\} \neq 0, N / 2$. By Lemma 2 ,

$$
L_{r}=\frac{\zeta^{\mu(2 c) 2 d}}{\zeta^{\mu(r c) r d}}
$$

thus $\left|C_{5}\right|=1$. However similarly we can show this equation is impossible. Hence at last we obtain $c \equiv 0 \bmod N$.

Now we show $d \equiv \pm 1 \bmod N$. By Proposition $1, W_{3}$ (resp. $W_{5}$ ) has poles only at the cusps $(u, t)$ such that $2 N / 5 \leq t \leq N / 2(\operatorname{resp} .3 N / 7 \leq$ $t \leq N / 2)$ and the order of the pole at $(u, t), t \neq N / 2$, is $(5 t-2 N) / D$ (resp. $(7 t-3 N) / D)$, while the order of the pole at $(u, N / 2)$ is 1 . Note that the order is determined only by $t$ and is independent of $u$. Thus we denote by $\nu_{r}\left(W_{r}\right)$ the order of the pole of the function $W_{r}$ at the cusp $(u, t)$.

If $N$ is odd (resp. $N \equiv 0 \bmod 4)$, then we see at once that $\nu_{r}(t)$ has the maximal value only at the cusp $\left(1, t_{0}\right)$, where $t_{0}=(N-1) / 2$ (resp. $N / 2-1)$. Since $c \equiv 0 \bmod N$, the matrix $A$ transforms a cusp $(u, t)$ to a cusp $(*,\{d t\})$. Therefore $d t_{0} \equiv \pm t_{0} \bmod N$. This shows that $d \equiv \pm 1 \bmod N$ and $A \in\{ \pm 1\} \Gamma_{1}(N)$.

Let $N \equiv 2 \bmod 4$. Then $\nu_{r}(t)$ takes the maximal value at the cusp $(1, N / 2-1)$ or $(1, N / 2-2)$. We must compare $\nu_{r}(N / 2-1)$ with $\nu_{r}(N / 2-2)$. If $r=3$ (resp. $r=5$ ), then $\nu_{r}(N / 2-2)>\nu_{r}(N / 2-1)$ if and only if $N>30$ (resp. $N>42$ ). Thus $d(N / 2-2) \equiv \pm(N / 2-2) \bmod N$ if $N>30$ (resp. $N>42)$, and $d(N / 2-1) \equiv \pm(N / 2-1) \bmod N$ if $N<30($ resp. $N<42)$. The former implies $d \equiv \pm 1 \bmod N$. The latter implies $d \equiv \pm 1 \bmod N / 2$ but since $d$ is odd, we know $d \equiv \pm 1 \bmod 2$, hence $d \equiv \pm 1 \bmod N$.

If $r=3, N=30$ or $r=5, N=42$, then $\nu_{r}(N / 2-2)=\nu_{r}(N / 2-1)$ and one of the following congruences holds true: $d(N / 2-2) \equiv \pm(N / 2-2) \bmod N$, $d(N / 2-1) \equiv \pm(N / 2-1) \bmod N, d(N / 2-2) \equiv \pm(N / 2-1) \bmod N$. The third congruence is impossible because $N, N / 2-1$ are even and $d, N / 2-2$ are odd. Hence also in this case $d \equiv \pm 1 \bmod N$.

Acknowledgements. The authors would like to express their hearty thanks to the referee for valuable suggestions and comments.

\section{References}

[1] J. W. S. Cassels, Local Fields, London Math. Soc. Stud. Texts 3, Cambridge Univ. Press, London, 1986.

[2] H. Darmon, Notes on a polynomial of Emma Lehmer, Math. Comp. 56 (1991), 795-800.

[3] N. Ishida, Generators and equations for modular function fields of principal congruence subgroups, Acta Arith. 85 (1998), 197-207.

[4] N. Ishida and N. Ishii, The equations for modular function fields of principal congruence subgroups of prime level, Manuscripta Math. 90 (1996), 271-285. 
[5] N. Ishida and N. Ishii, The equation for the modular curve $X_{1}(N)$ derived from the equation for the modular curve $X(N)$, Tokyo J. Math. 22 (1999), 167-175.

[6] D. Kubert and S. Lang, Units in the modular function fields, Math. Ann. 218 (1975), 175-189.

[7] O. Lecacheux, Unité d'une famille de corps cycliques réels de degré 6 lié à la courbe modulaire $X_{1}(13)$, J. Number Theory 31 (1989), 54-63.

[8] A. Ogg, Rational points on certain elliptic modular curves, in: Proc. Sympos. Pure Math. 24, Amer. Math. Soc., 1973, 221-231.

[9] M. A. Reichert, Explicit determination of nontrivial torsion structures of elliptic curves over quadratic number fields, Math. Comp. 46 (1986), 637-658.

[10] A. Robert, Elliptic Curves, Lecture Notes in Math. 326, Springer, 1973.

[11] L. C. Washington, A family of cyclic quartic fields arising from modular curves, Math. Comp. 196 (1991), 763-775.

Nobuhiko Ishida

Sanpo jyuutaku

2-4-9, Shinonome-higashi-machi

Sakai, Osaka 591-8041, Japan

E-mail: ishida@an.email.ne.jp
Noburo Ishii

Department of Mathematics and Information Sciences

College of Integrated Arts and Sciences

Osaka Prefecture University

1-1 Gakuen-cho

Sakai, Osaka 599-8531, Japan

E-mail: ishii@mi.cias.osakafu-u.ac.jp

Received on 10.4.2000

and in revised form on 25.4.2001 